\title{
Correction: Altered gut metabolome contributes to depression-like behaviors in rats exposed to chronic unpredictable mild stress
}

Li Jianguo (1) ${ }^{1,2}$, Jia Xueyang ${ }^{2,3}$, Wang Cui ${ }^{2,3}$, Wu Changxin ${ }^{1,2}$ and Qin Xuemei ${ }^{2,3}$

\section{Correction to: Translational Psychiatry \\ https://doi.org/10.1038/s41398-019-0391-z \\ published online January 2019}

After publication, the author's noted that the original Article contained clerical errors in the Results section.

The following passage from the original text:

"For example, we observed significant differences in body weight (Fig. 1c), most OFT indices (time spent immobile (Fig. 1d)), crossing counts (Fig. 1e), and rearing counts (Fig. 1f) starting in the 3rd week of exposure to CUMS. We did not observe significant changes in sucrose preference rate (Fig. 1b) or grooming time of OFT (Fig. 1g) until the last week of CUMS modeling, although an isolated notable change was evident in the 1st week."
Has been updated to:

"For example, we observed significant differences in body weight (Fig. 1c), two OFT indices (time spent immobile (Fig. 1d)), and rearing counts (Fig. 1f) starting in the 3rd week of exposure to CUMS. We did not observe significant changes in sucrose preference rate (Fig. 1b), grooming time (Fig. 1e) or crossing counts (Fig. 1g) of OFT until the last week of CUMS modeling, although an isolated notable change was evident in grooming time (Fig. 1e) at the 1st week."

Published online: 07 November 2019

Correspondence: Li Jianguo (lijg@sxu.edu.cn) or Qin Xuemei (qinxm@sxu.edu.cn) 'Laboratory of Microbiome and Health, Institutes of Biomedical Sciences, Shanxi University, Taiyuan 030006, China

2Key Laboratory of Chemical Biology and Molecular Engineering of Ministry of Education, Shanxi University, Taiyuan 030006, China

${ }^{3}$ Modern Research Center for Traditional Chinese Medicine, Shanxi University,

Taiyuan 030006, China

\section{(c) The Author(s) 2019}

(c) (i) Open Access This article is licensed under a Creative Commons Attribution 4.0 International License, which permits use, sharing, adaptation, distribution and reproduction (c) in any medium or format, as long as you give appropriate credit to the original author(s) and the source, provide a link to the Creative Commons license, and indicate if changes were made. The images or other third party material in this article are included in the article's Creative Commons license, unless indicated otherwise in a credit line to the material. If material is not included in the article's Creative Commons license and your intended use is not permitted by statutory regulation or exceeds the permitted use, you will need to obtain permission directly from the copyright holder. To view a copy of this license, visit http://creativecommons.org/licenses/by/4.0/. 